\title{
Design of Optimized Convolutional and Serially Concatenated Convolutional Codes in the Presence of A-priori Information
}

\author{
Andrea Abrardo, Member, IEEE, and Gianluigi Ferrari, Member, IEEE
}

\begin{abstract}
In this paper, we focus on the design of optimized binary convolutional codes (CCs) and serially concatenated convolutional codes (SCCCs) in the presence of a-priori information (API) at the receiver. For large signal-to-noise ratios (SNRs), we first propose a $\mathrm{CC}$ design criterion based on the minimization of a union bound on the bit error probability (BEP). In this case, relevant performance gains, with respect to previously proposed CCs, are obtained. These gains persist even in the presence of estimation errors on the API. Then, we apply the same union bound-based design criterion to SCCCs. Since the BEP of SCCCs is characterized by a typical waterfall shape, the proposed union bound-based design criterion is accurate only at large SNR, to estimate the BEP floor. In order to complement this analysis, we propose a density evolution-based approach to optimize the SCCC design in terms of minimization of the SNR of the "knee" of the BEP curve. The obtained simulation results show substantial gains with respect to previously proposed parallel concatenated convolutional coding (PCCCing) schemes optimized under the assumption of no API at the decoder. Moreover, in the presence of strong API the proposed SCCCs allow to approach the Shannon limit (SL) more than any previously proposed turbo coding scheme.
\end{abstract}

Index Terms-Convolutional codes (CCs), serially concatenated convolutional codes (SCCCs), a priori information.

\section{INTRODUCTION}

$\mathbf{I}$ $\mathrm{N}$ most binary digital communication systems with binary coding at the information sources, the bits of the sequence at the output of a source encoder are assumed to be independent and identically distributed (with uniform distribution). However, the majority of existing source encoders-in particular, fixed-length encoders commonly used for transmission over noisy channels-do not respect this condition. More precisely, a sequence output by a source encoder contains some residual redundancy. Therefore, reliable transmission of coded sequences with redundancy (residual, if source coding is considered; natural, otherwise) is an important issue [1]. In this case, the performance of the channel decoder can be improved if the redundancy at the output of the encoder is properly exploited.

Manuscript received February 10, 2010; revised April 27, 2010; accepted October 20,2010. The associate editor coordinating the review of this paper and approving it for publication was M. Ardakani.

A. Abrardo is with the Department of Information Engineering, University of Siena, Via Roma 56, 53100 Siena, Italy (e-mail: abrardo@dii.unisi.it).

G. Ferrari is with the WASN Lab, Department of Information Engineering, University of Parma, Viale Usberti 181/A, 43124 Parma, Italy (e-mail: gianluigi.ferrari@unipr.it).

Digital Object Identifier 10.1109/TWC.2010.120310.100153
A typical scenario where some residual (or total) redundancy is left after source encoding is represented by the transmission of sources with memory. In this case, the problem of exploiting the source memory at the channel decoder is often referred to as joint source-channel decoding (JSCD) problem. One of the main limitations for the design of JSCD schemes is represented by the implementation complexity of the decoder, which tends to become unmanageable if the memory of the information source is taken into account. For example, in the presence of a first-order Markov source followed by a convolutional code (CC), the optimum JSCD scheme is given by the use, at the receiver, of a maximum a posteriori (MAP) sequence decoder based on a "super-trellis" diagram, whose number of states is given by the product of the number of states of the $\mathrm{CC}$ and the number of states of the Markov source. A few methods have been proposed to reduce the number of states of the super-trellis diagram, thus leading to suboptimal MAP decoders [1]-[6]. Rather than reducing the complexity of super-trellis-based sequence decoders, another approach to perform JSCD consists in exploiting the redundancy of the information source as a-priori information (API) at the input of channel decoder/demodulator. In this case, one can derive iterative decoding schemes, where, at each iteration, the API is taken into account by the decoder without substantially increasing the overall receiver complexity. In particular, when bit-level API is considered, the derived iterative channel decoding schemes can be straightforwardly applied in the presence of turbo ${ }^{1}$

Another application scenario where JSCD is gaining its momentum is the transmission of signals observed at different nodes, such as in wireless sensor networks (WSNs) [9]. In this context, several papers have proposed JSCD schemes where correlated sources are independently channel encoded at a reduced rate (with respect to the uncorrelated case). The reduced transmission reliability, due to channel coding rate reduction, can then be compensated, at the receiver, by exploiting the existing correlation among different information sources [10]-[14]. In this case, the receiver makes use of an iterative decoder which contains one component subdecoder per information source. The source correlation is exploited by iteratively exchanging (properly updated) API between the various subdecoders.

Although the gains achievable by the use of channel coding

\footnotetext{
${ }^{1}$ In the literature, the general term "turbo code" refers to either a parallel concatenated convolutional code (PCCC) or a serially concatenated convolutional code (SCCC).
} 
in the presence of API are considerable, in the literature the performance gaps, with respect to the Shannon limit (SL), are still relatively large, even if a significant API is available. On the other hand, in [15] it is shown that the empirical distribution of the sequences at the output of the encoder of a good code (i.e., a code with vanishing probability of error, for sufficiently long blocklengths, at a rate close to the SL) should approach the capacity-achieving input distribution. Hence, since the presence of API at the decoder can be equivalently interpreted as the presence of nonuniform input information sequences at the encoder, it is expected that a good binary code for effective decoding in the presence of API should be designed to produce uniform, independent, and identically distributed output bits in the presence of nonuniform input binary sequences.

To the best of our knowledge, the only paper which investigates the problem of designing good codes for decoding with API (or, equivalently, with nonuniform input information sequences) is [16]. In particular, in [16] it is shown that using recursive nonsystematic $\mathrm{CCs}$ as component codes of a parallel concatenated convolutional code (PCCC) leads to asymptotically uniformly distributed output sequences even in the presence of strongly non-uniformly distributed input sequences. Accordingly, the proposed PCCC structure guarantees substantial gains over previously designed turbo and LDPC codes.

In this paper, we propose simple criteria for the design of good codes in the presence of API at the receiver. In particular, our first design criterion aims at the minimization of an analytically derived union bound on the bit error probability (BEP). The proposed framework is first applied to CCs. As in [16], we show that recursive nonsystematic CCs are to be preferred in the presence of strong API. The same BEPbased optimization criterion is applied to SCCCs. In this case, simulation results, relative to SCCCs designed according to the proposed BEP-based criterion, show substantial gains with respect to turbo coding schemes optimized in the absence of API at the decoder. Unlike the BEP of CCs, the BEP of SCCCs is characterized by a typical "waterfall" shape with a floor at its bottom: the proposed BEP union bound-based code optimization approach applies only at large SNRs, i.e., in correspondence to the BEP floor. In order to complement it, we derive a density evolution-based design criterion which aims at minimizing the SNR of the "knee" of the BEP curve, i.e., is valid at low SNRs. The obtained density evolutionbased results compare favorably with the union bound-based optimization results. In particular, we design a rate-1/2 SCCC which, in the presence of strong API, allows to approach the SL more than any previously proposed turbo code (for instance, the PCCCs proposed in [16]).

This paper is structured as follows. In Section II, we present the coded communication scenario considered in the remainder of the paper. In Section III, we derive a BEP-based analytical framework for the design of good CCs and SCCCs for large values of the SNR. In Section IV, we propose a density evolution-based approach to the design of SCCCs and, as a relevant case study, we focus on rate-1/2 SCCCs. Finally, concluding remarks are given in Section V.

\section{Coded Communication Scenario}

We consider an independent and identically distributed (i.i.d.) binary source sequence $\boldsymbol{x}$ of length $k$ which is channel encoded with rate $r=k / n$ and transmitted into a binary Gaussian channel. The binary coded signal of length $n$ is denoted as $\boldsymbol{c}$. We assume that side-information, i.e., API, on the information sequence $\boldsymbol{x}$, in terms of estimated bit sequence $\tilde{x}$, where $\tilde{x}_{i} \in\{0,1\}, i=1, \ldots, k$, is available at the receiver side. We denote as $\rho$ the bit reliability of the API, i.e.,

$$
\rho \triangleq \operatorname{Pr}\left(\tilde{x}_{i}=x_{i}\right) \quad \forall i
$$

and we introduce the following a-priori log-likelihood ratios (LLRs):

$$
L\left(x_{i}\right) \triangleq \ln \left[\frac{\operatorname{Pr}\left(x_{i}=0\right)}{\operatorname{Pr}\left(x_{i}=1\right)}\right] \quad \forall i
$$

Given the above notations, it is easy to show that

$$
L\left(x_{i}\right)=L(\rho)\left(-2 \tilde{x}_{i}+1\right)
$$

where

$$
L(\rho) \triangleq \ln \left(\frac{\rho}{1-\rho}\right) .
$$

In order to fruitfully exploit the API, the channel decoder must estimate $\rho$. In the following, since the focus of this work is not on the derivation of accurate estimation strategies for $\rho$, we simply assume that an estimate $\tilde{\rho}$ is available at the decoderthe reader is referred to existing works in the literature, such as [13], for more details on possible estimation strategies. The estimated reliability of an information bit is thus $L(\tilde{\rho})$.

Let us now denote by $\boldsymbol{x}_{\text {est }} \neq \boldsymbol{x}$ the sequence estimated through MAP decoding. Moreover, denote by $\boldsymbol{c}_{\text {est }} \neq \boldsymbol{c}$ the corresponding codeword. The evaluation of the probability of estimation error has been recently investigated in [17]. In particular, in [17] the following exact expression for the pairwise error probability (PEP), i.e., the probability that the decoder detects $\boldsymbol{x}_{\text {est }}$ instead of $\boldsymbol{x}$, is derived in a scenario with binary phase-shift keying (BPSK) (or binary pulse amplitude modulation, PAM):

$$
\begin{aligned}
P_{\mathrm{e}, \mathrm{pair}}(d, w)= & \frac{1}{2} \sum_{\tilde{w}=0}^{w} \operatorname{erfc}\left(\sqrt{r d \gamma_{\mathrm{b}}\left(1+\frac{L(\tilde{\rho})(w-2 \tilde{w})}{4 d r \gamma_{\mathrm{b}}}\right)^{2}}\right) \\
& \times\left(\begin{array}{c}
w \\
\tilde{w}
\end{array}\right) \rho^{w-\tilde{w}}(1-\rho)^{\tilde{w}}
\end{aligned}
$$

where: $\operatorname{erfc}(x) \triangleq(2 / \sqrt{\pi}) \int_{x}^{\infty} e^{-t^{2}} \mathrm{~d} t ; w \triangleq D\left(\boldsymbol{x}, \boldsymbol{x}_{\text {est }}\right)=\sum_{i=1}^{k} x_{i} \oplus$ $x_{\text {est }, i}$ is the Hamming distance between $\boldsymbol{x}_{\text {est }}$ and $\boldsymbol{x} ; \boldsymbol{d} \triangleq$ $D\left(\boldsymbol{c}, \boldsymbol{c}_{\text {est }}\right)$ is the Hamming distance between $\boldsymbol{c}$ and $\boldsymbol{c}_{\text {est }}$; and $\gamma_{\mathrm{b}} \triangleq E_{\mathrm{b}} / N_{0}$, where $E_{\mathrm{b}}$ is the average energy per source bit and $N_{0}$ is the monolateral power spectral density of the channel additive white Gaussian noise. As shown in [17], by properly manipulating (2) it is possible to derive the following upper bound:

$$
P_{\mathrm{e}, \mathrm{pair}}(d, w) \leq e^{-r d \gamma_{\mathrm{b}}} A^{w}
$$

where

$$
A=A(\rho, \tilde{\rho}) \triangleq(1-\rho) \sqrt{\frac{\tilde{\rho}}{1-\tilde{\rho}}}+\rho \sqrt{\frac{1-\tilde{\rho}}{\tilde{\rho}}} .
$$




\section{HIGH SNR: BEP UNION BOUND-BASED DESIGN OF CCS AND SCCCS}

\section{A. Convolutional Codes}

Convolutional coding allows an easy coding implementation, with very low power and memory requirements [18], [19]. Therefore, this type of channel coding seems to be particularly attractive for WSN applications. Moreover, if MAP sequence decoding is considered in the presence of API, optimal decoding algorithms can be straightforwardly derived by properly taking the API into account in the branch metrics of a Viterbi algorithm.

In convolutional decoding without API, it is possible to derive an upper bound on the BEP as the weighed ${ }^{2}$ sum of the PEPs associated to all trellis paths which diverge from the all zero-state path (associated with the all-zero information sequence) and, later, merge again with the latter [18]. This approach is applicable also in the presence of API, owing to the linearity of the code and to the fact that the PEP (2) depends only on the weights $d$ and $w$, but not on the actual transmitted sequence. In particular, it is possible to evaluate the input-output transfer function $T(W, D)$ by means of the state transition relations over the modified state diagram associated with the used CC [18]. The generic form of the transfer function is the following:

$$
T(W, D)=\sum_{w, d} \beta_{w, d} W^{w} D^{d}
$$

where $\beta_{w, d}$ denotes the number of paths that temporarily diverge from the zero-state path and are associated with an input (information) sequence of weight $w$ and with an output (coded) sequence of weight $d$. Accordingly, an upper bound on the BEP is

$$
P_{\mathrm{e}, \mathrm{b}} \leq \sum_{w, d} \beta_{w, d} w P_{\mathrm{e}, \mathrm{pair}}(d, w)
$$

where $P_{\mathrm{e}, \text { pair }}(d, w)$ has the expression (2). A looser upper bound can be derived by applying the upper bound (3) in (6), thus obtaining:

$$
P_{\mathrm{e}, \mathrm{b}} \leq \sum_{w, d} \beta_{w, d} w e^{-r d \gamma_{\mathrm{b}}} A^{w}
$$

From (5) and (7), it is straightforward to rewrite (7) as follows:

$$
P_{\mathrm{e}, \mathrm{b}} \leq\left. A \frac{\partial T(W, D)}{\partial W}\right|_{W=A, D=e^{-r \gamma_{\mathrm{b}}}} .
$$

At this point, a CC design criterion consists in minimizing (7) (or, equivalently, (8)). At large SNRs, at the right-hand side of (7) only the terms with $d=d_{\text {free }}$ are relevant, i.e., one can write:

$$
P_{\mathrm{e}, \mathrm{b}} \lesssim \sum_{w} \beta_{w, d_{\text {free }}} w e^{-r d_{\text {free }} \gamma_{\mathrm{b}}} A^{w}=e^{-r d_{\text {free }} \gamma_{\mathrm{b}}} \sum_{w} \beta_{w, d_{\text {free }}} w A^{w} .
$$

The design criterion thus reduces to search, among the set of maximum free-distance codes, the code which minimizes the term $\sum_{w} \beta_{w, d_{\text {free }}} w A^{w}$. Obviously, this optimization depends on the value of $A$ (i.e., on $\rho$ and $\tilde{\rho}$ ) and on the input-output weight enumerator coefficients $\left\{\beta_{w, d_{\text {free }}}\right\}$, which depend on the encoder structure. Therefore, assuming $\rho=\tilde{\rho}$ (from (4),

\footnotetext{
${ }^{2}$ The weights are information error weights.
}

TABLE I

OPTIMIZED FEEDFORWARD $\left(G^{(1)}, G^{(2)}\right)$ AND FEEDBACK $(H)$ GENERATOR POLYNOMIALS OF RATE- $1 / 2$ CCS AS FUNCTIONS OF $\rho$ AND $v$. IN ALL CASES, $\tilde{\rho}=\rho$.

\begin{tabular}{|c|c|c|c|c|}
\hline$\rho$ & $v$ & $G^{(1)}$ & $G^{(2)}$ & $H$ \\
\hline 0.5 & 2 & 5 & 7 & 1 \\
\hline 0.6 & 2 & 5 & 7 & 1 \\
\hline 0.7 & 2 & 5 & 7 & 1 \\
\hline 0.8 & 2 & 5 & 7 & 1 \\
\hline 0.9 & 2 & 5 & 7 & 1 \\
\hline 0.95 & 2 & 5 & 7 & 7 \\
\hline 0.5 & 3 & 15 & 17 & 1 \\
\hline 0.6 & 3 & 15 & 17 & 1 \\
\hline 0.7 & 3 & 15 & 17 & 1 \\
\hline 0.8 & 3 & 15 & 17 & 1 \\
\hline 0.9 & 3 & 15 & 17 & 11 \\
\hline 0.95 & 3 & 15 & 17 & 11 \\
\hline 0.5 & 4 & 23 & 35 & 1 \\
\hline 0.6 & 4 & 23 & 35 & 1 \\
\hline 0.7 & 4 & 23 & 35 & 1 \\
\hline 0.8 & 4 & 23 & 35 & 1 \\
\hline 0.9 & 4 & 23 & 35 & 37 \\
\hline 0.95 & 4 & 23 & 35 & 37 \\
\hline 0.5 & 5 & 53 & 75 & 1 \\
\hline 0.6 & 5 & 53 & 75 & 1 \\
\hline 0.7 & 5 & 53 & 75 & 1 \\
\hline 0.8 & 5 & 53 & 75 & 1 \\
\hline 0.9 & 5 & 53 & 75 & 63 \\
\hline 0.95 & 5 & 53 & 75 & 63 \\
\hline
\end{tabular}

$A=2 \sqrt{\rho(1-\rho)}$ ) and a code rate equal to $1 / 2$, for given values of $\rho$ and of the number $v$ of the shift registers of the convolutional encoder (i.e., the number of states of the $\mathrm{CC}$ is $2^{v}$ ), we determine, through an exhaustive search, the best encoder structure, identified by two feedforward generator polynomials (denoted as $G^{(1)}$ and $G^{(2)}$ ) and one feedback polynomial (denoted as $H$ ). The obtained results are shown in Table I (the polynomials are expressed in octal notation). As one can see, for large values of the API $(\rho \geq 0.95$ for $v=2$ and $\rho \geq 0.9$ for $v \geq 3$ ) the optimized CCs become recursive, whereas for smaller values of $\rho$ the optimized CCs are the standard non-recursive ones (for example, for $v=3$ the standard $(15,17) \mathrm{CC}$ is obtained [19]). Since the feedforward polynomial generators remain the same, regardless of the value of $\rho$, it follows that code optimization reduces to encoder optimization. In other words, while the codebook remains the same (i.e., $d_{\text {free }}$ is the same), regardless of the value of $\rho$, the mapping between the information sequences and the codewords has to be optimized. In the absence of API (i.e., $\rho=0.5$ and, thus, $A=1$ ), the optimization procedure reduces to the optimization of the input-output weight enumerator coefficients $\left\{\beta_{w, d_{\text {free }}}\right\}$ [20], [21].

In order to provide a rationale for the fact that recursive encoders are to preferred, with respect to non-recursive ones, for high values of $\rho$, we refer to the case with $v=3$ and investigate the transfer functions of the optimized codes. The optimized CC for $\rho=0.5$, denoted as $\mathscr{C}_{50}$, is the non-recursive convolutional code (NRCC) $(15,17)$, whereas the optimized CC for $\rho=0.9$, denoted as $\mathscr{C}_{90}$, is the recursive convolutional code (RCC) $(15,17,11)$. Since the transfer function of $\mathscr{C}_{50}$ is 


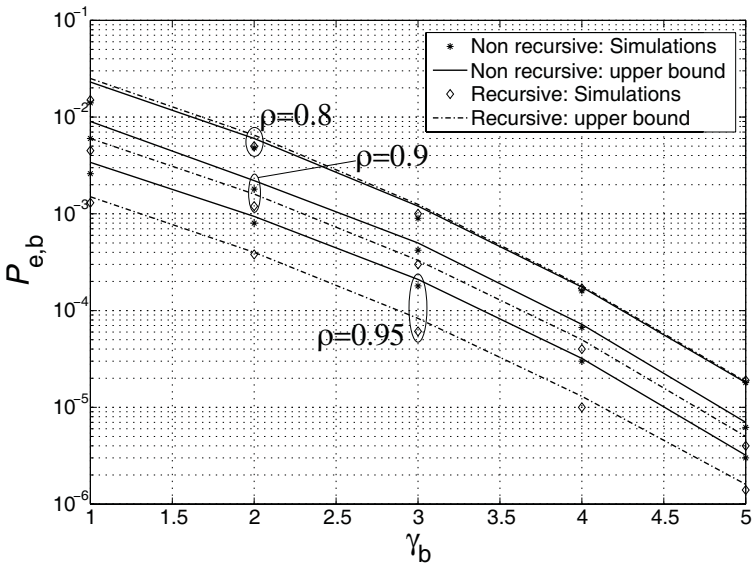

Fig. 1. Comparative evaluation of the BEP, as a function of the SNR, with the RCC $\mathscr{C}_{90}$ and the NRCC $\mathscr{C}_{50}$. Simulation results (lines) are shown together with the the corresponding upper bounds given by (6) (symbols). Three different values for $\rho=\tilde{\rho}$ are considered, namely $0.8,0.9$, and 0.95 .

the following:

$$
T(D, W)=\frac{D^{6} W^{2}+D^{7} W-D^{8} W^{2}}{1-2 D W-D^{3} W}=D^{6} W^{2}+D^{7} W+\ldots
$$

it can be concluded that the minimum distance (for the coded sequence) $d_{\text {free }}$ is 6 and the corresponding information weight is 2 . In the case of $\mathscr{C}_{90}$, it is possible to verify that the transfer function is characterized by a minimum distance $d_{\text {free }}$ equal to 6 , with corresponding information weight equal to 4 . Hence, considering the upper bound (7), for high channel SNRs (when only the minimum distance term at the right-hand side of (7) is non-negligible) the BEP guaranteed by the two optimum CCs can be approximately upper bounded as follows:

$$
P_{\mathrm{e}, \mathrm{b}} \lesssim \begin{cases}A^{2} \operatorname{erfc}\left(\sqrt{3 \gamma_{\mathrm{b}}}\right) & \text { for } \mathscr{C}_{50} \\ 2 A^{4} \operatorname{erfc}\left(\sqrt{3 \gamma_{\mathrm{b}}}\right) & \text { for } \mathscr{C}_{90} .\end{cases}
$$

Therefore, we expect that the RCC $\mathscr{C}_{90}$ will outperform the NRCC $\mathscr{C}_{50}$ when $A<1 / \sqrt{2}$. Under the assumption of perfect reliability estimation, i.e., $\rho=\tilde{\rho}$, this means that the RCC is expected to perform better for $\rho>0.85$.

The validity of the previous analytical code design framework can be assessed by means of computer simulations. In the first set of simulations, we have compared the BEPs of $\mathscr{C}_{90}$ and $\mathscr{C}_{50}$ assuming perfect reliability estimation, i.e., $\rho=\tilde{\rho}$. The obtained results are shown in Fig. 1 for different values of $\rho$, namely, $0.8,0.9$, and 0.95 . In all cases, the BEP curves relative to $\mathscr{C}_{50}$ are labelled as "non recursive," whereas the BEP curves relative to $\mathscr{C}_{90}$ are labeled as "recursive." Moreover, each simulation-based curve is shown together with the corresponding upper bound given by (6). As one can observe, the analytical upper bound on the BEP is quite tight and tends to perfectly match the simulation results for high values of the SNR. As expected, the RCC clearly outperforms the NRCC for $\rho \geq 0.9$. On the other hand, for $\rho=0.8$ the performance of the two CCs is basically the same.

It is then of interest to investigate the effect of imperfect reliability estimation, i.e., the case with $\rho \neq \tilde{\rho}$. A relevant performance indicator is given by the channel SNR required to

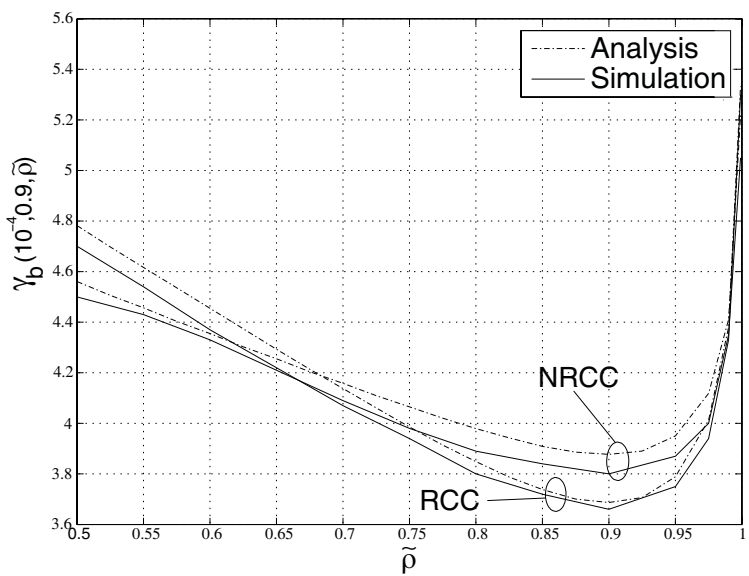

Fig. 2. The channel SNR $\gamma_{\mathrm{b}}\left(10^{-4}, 0.9, \tilde{\rho}\right)$ is shown as a function of $\tilde{\rho}$. Simulation results (solid lines) and analytical results based on (11) (dashed lines) are shown, considering the RCC $\mathscr{C}_{90}$ and the NRCC $\mathscr{C}_{50}$.

achieve a given BEP $P_{\mathrm{e}, \mathrm{b}}$. We denote this $\operatorname{SNR}$ as $\gamma_{\mathrm{b}}\left(P_{\mathrm{e}, \mathrm{b}}, \rho, \tilde{\rho}\right)$, to underline that it corresponds to the channel SNR at which a BEP $P_{\mathrm{e}, \mathrm{b}}$ is achieved in a scenario where $\rho$ is the true API and $\tilde{\rho}$ is the estimated value used by the receiver. This channel SNR can be evaluated, through simulations, as the value at which the target BEP $P_{\mathrm{e}, \mathrm{b}}$ is achieved. On the other hand, it can also be approximated by relying on the previous analysis. To this aim, the upper bound (6) can be used as an estimate, denoted as $\tilde{P}_{\mathrm{e}, \mathrm{b}}$, of the BEP in the presence of imperfect reliability estimation, i.e.,

$$
\tilde{P}_{\mathrm{e}, \mathrm{b}} \triangleq \sum_{w, d} \beta_{w, d} w P_{\mathrm{e}, \mathrm{pair}}(d, w) .
$$

The channel SNR $\gamma_{\mathrm{b}}\left(\tilde{P}_{\mathrm{e}, \mathrm{b}}, \rho, \tilde{\rho}\right)$ can be numerically determined from (11). In Fig. 2, $\gamma_{\mathrm{b}}\left(P_{\mathrm{e}, \mathrm{b}}, \rho, \tilde{\rho}\right)=\gamma_{\mathrm{b}}\left(10^{-4}, 0.9, \tilde{\rho}\right)$ is shown as a function of $\tilde{\rho}$, considering the RCC $\mathscr{C}_{90}$ and the NRCC $\mathscr{C}_{50}$. Simulation results are shown as solid lines, whereas analytical results are shown as dashed lines. Obviously, in each case the best performance, i.e., the lowest value of $\gamma_{b}$, is achieved when $\tilde{\rho}=\rho$. It can be observed that the RCC $\mathscr{C}_{90}$ takes greater advantage, with respect to the NRCC $\mathscr{C}_{50}$, from the use of API. For instance, the maximum SNR gain (obviously achieved when $\tilde{\rho}=\rho$ ) is $1 \mathrm{~dB}$ for the RCC and $0.7 \mathrm{~dB}$ for the NRCC. On the other hand, the RCC is more sensitive to underestimation of the API: this is due to the higher information weight associated with the free distance term in the transfer function. Finally, for values of $\tilde{\rho}$ close to 1 , i.e., for $\tilde{\rho}>\rho$, it can be observed that the required channel SNR increases significantly, regardless of the code: this suggests that the overestimation of $\rho$ has a much more detrimental impact than its underestimation.

\section{B. Serially Concatenated Convolutional Codes}

A SCCC consists of a cascade of two bit-interleaved component codes, namely an outer $\mathrm{CC}$ and an inner $\mathrm{CC}$ [22]. The corresponding decoder relies on a message passing algorithm, such that extrinsic information is iteratively passed, for a predefined number of iterations, between the inner and outer soft-input soft-output (SISO) decoders, corresponding to the 
inner and outer CCs. The presence of API (relative to the information bits at the input of the outer CC) can be easily taken into account by transforming the a priori probabilities of information bits into LLRs and feeding the outer SISO decoder with them (together with the LLRs generated by the inner SISO decoder). Hence, the SISO outer decoder completely exploits the API (if present), whereas the inner decoder works as in traditional SCCC decoders without API.

In [22], upper bounds on the average BEP of SCCCs under maximum likelihood decoding are derived. The approach followed in [22] is based on the derivation of the input-output weight enumerating function (IOWEF) for the equivalent block code which represents the cascade of the component codes of the SCCC. In particular, the IOWEF for the SCCC can be expressed as

$$
Q(W, H)=\sum_{w, h} Q_{w, h} W^{w} H^{h}
$$

where $Q_{w, h}$ represents the number of codewords with weight $h$ generated by information words of weight $w$ and can be given the following expression:

$$
Q_{w, h}=\sum_{\ell=0}^{N} \frac{Q_{w, \ell}^{(\mathrm{o})} Q_{\ell, h}^{(\mathrm{i})}}{\left(\begin{array}{c}
N \\
\ell
\end{array}\right)}
$$

where $N$ is the interleaver length and $Q_{w, \ell}^{(\mathrm{o})}$ and $Q_{\ell, h}^{(\mathrm{i})}$ are the coefficients $^{3}$ of the IOWEFs of the outer and inner CCs, respectively. Leveraging on the derivation in the previous subsection, the BEP under maximum likelihood decoding in the presence of API can be upper bounded as:

$$
P_{\mathrm{e}, \mathrm{b}} \leq \sum_{w, h} Q_{w, h} w A^{w} e^{-r h \gamma_{\mathrm{b}}}
$$

where $r=r^{(\mathrm{o})} r^{(\mathrm{i})}$ is the code rate of the SCCC, $r^{(\mathrm{o})}$ and $r^{(\mathrm{i})}$ being the outer and the inner CCs' rates, respectively.

In order to carry out preliminary design considerations for the SCCCs, we refer to the conclusions drawn in [22] for a scenario with no API. In particular, in [22] it is shown that an interleaver gain, i.e., the BEP is an increasing function of the interleaving length $N_{\text {int }}$, is achievable provided that the inner CC is recursive. It is straightforward to observe that the presence of API does not affect this conclusion and, hence, also in presence of API it can be concluded that the inner CC has to be recursive. In particular, we limit ourselves to the inner systematic RCCs derived from the equivalent NRCCs, with maximum free distance, shown in [19].

In order to derive design guidelines for the outer $\mathrm{CC}$, we rely on the analysis, proposed in [22], based on the maximum exponent of $N_{\text {int }}$. For the sake of notational simplicity, we consider the case with even minimum free distance of the outer CC, denoted as $d_{\mathrm{f}}^{(\mathrm{o})}$. In this case, from [22] and on the basis of the analysis presented in the previous subsection, the

\footnotetext{
${ }^{3}$ More precisely, $Q_{w, \ell}^{(\mathrm{o})}$ represents the number of codewords, with weight $\ell$, generated by information words of weight $w$ at the input of the outer decoder and $Q_{\ell, h}^{(\mathrm{i})}$ represents the number of codewords, with weight $h$, generated by information words of weight $\ell$ at the input of the inner decoder.
}

following upper bound on the BEP can be derived:

$$
P_{\mathrm{e}, \mathrm{b}} \leq K^{(\mathrm{o})} \frac{d_{\mathrm{f}}^{(\mathrm{o})_{\mathrm{f}}^{(\mathrm{o})}} d_{\mathrm{f}}^{(\mathrm{o})} !}{\left(d_{\mathrm{f}}^{(\mathrm{o})} / 2\right) !} N_{\mathrm{int}}^{\frac{-d_{\mathrm{f}}^{(\mathrm{o})}}{2}} N_{\mathrm{f}}^{(\mathrm{o})} w_{\min } A^{w_{\min }} e^{-r \frac{d_{\mathrm{f}}^{(\mathrm{o})} d_{\mathrm{f}}^{(\mathrm{i})}}{2} \gamma_{\mathrm{b}}}
$$

where: $K^{(\mathrm{o})}$ is a constant which depends on the CC structure; $w_{\min }$ is the minimum input weight yielding outer codewords with weight equal to $d_{\mathrm{f}}^{(\mathrm{o})} ; N_{\mathrm{f}}^{(\mathrm{o})}$ is the number of such codewords; and $d_{\mathrm{f}}^{(\mathrm{i})}$ is the minimum weight of codewords of the inner CC (generated by weight- 2 input information sequences). The upper bound (15) allows to draw important design guidelines for the outer CC. In particular, the outer $\mathrm{CC}$ must be designed to jointly maximize the minimum free distance and the minimum input information weight yielding outer codewords with weight $d_{\mathrm{f}}^{(\mathrm{o})}$. This design rule is coherent with the design rule of a CC in the presence of API and, hence, the outer decoder can be designed following the optimization criterion presented in Subsection III-A. More precisely, the performance is optimized when the outer $\mathrm{CC}$ is a nonsystematic RCC.

In order to verify and confirm the above SCCC design conjecture, we have computed the BEP upper bound (14) for two rate-1/4 SCCCs. In the first SCCC, referred to as "NRCC outer-RCC inner," the outer code is the rate-1/2 NRCC $\mathscr{C}_{50}$ and the inner code is the rate-1/2 systematic RCC with the following generators: $G^{(1)}=15, G^{(2)}=17$, and $H=15$. Note that the latter RCC is the systematic version of the NRCC $\mathscr{C}_{50}$, i.e., it is chosen according to the optimal design rule for the inner code. The second considered SCCC, referred to as "RCC outer-RCC inner," uses the same inner code as the second SCCC and the rate-1/2 RCC $\mathscr{C}_{90}$ as outer code. Note that this SCCC is derived by applying directly the optimization criterion presented in Subsection III-A. In other words, the "RCC outer-RCC inner" code design is coherent with the optimal design conjecture previously discussed. In all cases, the interleaver length $N_{\text {int }}$ is set to 200. In Fig. 3, the upper bound (14) on the BEP is shown, as a function of the SNR, for the two considered SCCCs. In each case, various values of $\rho$ (namely, 0.9,0.7, and 0.5) are considered and perfect reliability estimation (i.e., $\tilde{\rho}=\rho$ ) is assumed. It is worth noting that in the presence of significant API $(\rho=0.9)$, the "RCC outer-RCC inner" SCCC shows a clear advantage with respect to the other SCCC, thus confirming the validity of the optimized design conjecture. Moreover, in the presence of limited API, i.e., $\rho \leq 0.7$, the optimal choice is to use an outer NRCC and an inner RCC, which is consistent with the design rule derived in [22] for a scenario with no API.

\section{Low SNR: Density Evolution-BASEd Design of SCCCS}

The analysis in Subsection III-B is useful to analyze the performance of SCCCs in the error floor region. However, union bounds give little information on the BEP performance in the waterfall region. In this section, we propose a design criterion, using density evolution techniques, for SCCC optimization in the waterfall region. 


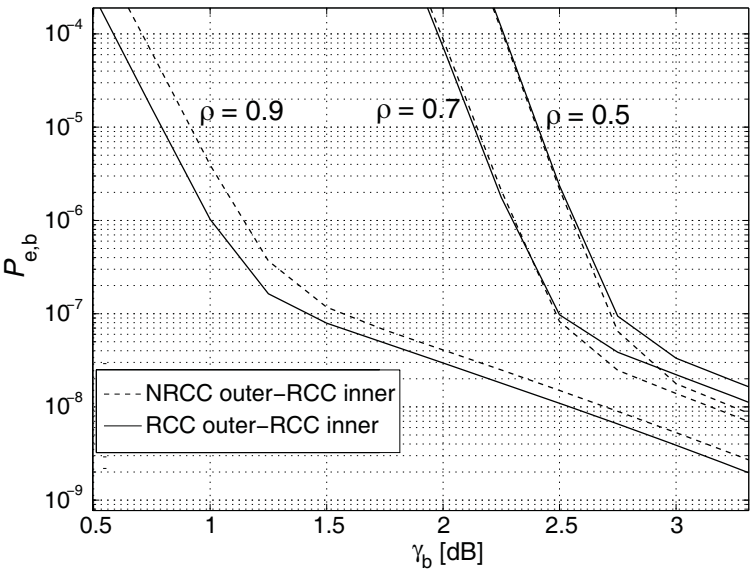

Fig. 3. Analytical BEP bounds, for different rate-1/4 SCCCs, in the presence of API.

\section{A. Preliminaries on Density Evolution}

We refer to the classical density evolution framework proposed in [23], properly adapted to the presence of API at the receiver. In Fig. 4, the reference scheme for density evolutionbased design of a SCCC in the presence of API is shown. In this figure, the blocks indicated with $\mathrm{DEC}^{(\mathrm{o})}$ and $\mathrm{DEC}^{(\mathrm{i})}$ correspond to the outer and inner decoders, respectively. We assume that the source transmits the all-zero sequence, i.e., $x_{i}=0, \forall i$. In the considered scenario, the messages (e.g., LLRs), derived from the channel observables and fed at the input of $\mathrm{DEC}^{(\mathrm{i})}$, are Gaussian random variables with probability density function (pdf) $\mathscr{N}\left(2 \gamma_{b}, \sqrt{4 \gamma_{b}}\right)$. For the purpose of density consistency, required for a density evolution-based analysis to be applicable [23], we assume that each internal message (at the input or output of a component decoder) is Gaussian as well, with pdf $\mathscr{N}(\mu, \sqrt{2 \mu})$ and corresponding SNR given by $\mu / 2$. In the considered scenario, however, the internal LLRs at the input of $\mathrm{DEC}^{(\mathrm{o})}$ might not be, in general, Gaussian. In the next paragraph, we describe how an approximating Gaussian distribution can then be determined and we evaluate its accuracy.

According to the considered API model and under the assumption of all-zero transmitted information sequence, it is straightforward to observe that the a-priori LLR for a bit of the information sequence ${ }^{4}$ can be expressed as follows:

$$
L(x)= \begin{cases}L(\tilde{\rho}) & \text { with probability } \rho \\ -L(\tilde{\rho}) & \text { with probability } 1-\rho\end{cases}
$$

where $L(\tilde{\rho})$ has been introduced in Section II. At this point, denoting as $a_{x}(z)$ ( $z$ is a dummy variable) the pdf of an a-priori LLR, one can write:

$$
a_{x}(z)=\rho \delta(z-L(\tilde{\rho}))+(1-\rho) \delta(z+L(\tilde{\rho}))
$$

where $\delta(\cdot)$ is the Dirac delta function. Remark, therefore, that the pdf $a_{x}(z)$ depends on the two parameters $\{\rho, \tilde{\rho}\}$. Given the above, the evolution of the pdfs of the messages at the input and output of the component decoders $\mathrm{DEC}^{(\mathrm{o})}$ and $\mathrm{DEC}^{(\mathrm{i})}$ in Fig. 4 can then be determined through very simple (and

\footnotetext{
${ }^{4}$ For ease of notational simplicity, we have dropped the subscript $i$ for $x$, since the expression of the pdf does not depend on the particular bit.
}

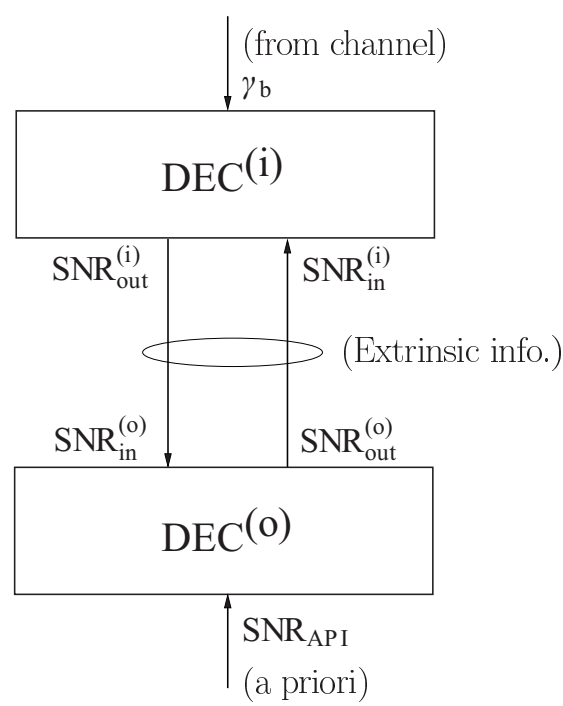

Fig. 4. Density evolution scheme for an SCCC in the presence of API.

accurate) simulations. More precisely, for each component decoder one can run a SISO decoding algorithm on a very long trellis, feeding it with independent (channel and a-priori) LLRs drawn according to corresponding input distributions characterized by the parameters $\gamma_{\mathrm{b}}$ and $\{\rho, \tilde{\rho}\}$, respectively. From the output LLRs, one can then estimate the output SNRs and derive a corresponding "forced" Gaussian pdf as follows. Owing to the assumption of all-zero transmitted sequence, the BEP at the output of the component decoder, denoted as $P_{\mathrm{e}, \text { out }}$, may be numerically evaluated from the output LLRs. Hence, by setting to $P_{\mathrm{e}, \text { out }}$ the probability that a Gaussian random variable (the output LLR) with distribution $N(\mu, \sqrt{2 \mu})$ is lower than zero, $\mu$ can be estimated as follows:

$$
\mu=4\left\{\operatorname{erfc}^{-1}\left[2 P_{\mathrm{e}, \mathrm{out}}\right]\right\}^{2} .
$$

The corresponding output SNR is then $\mu / 2$.

In order to assess the validity of the Gaussian approximation, as a meaningful illustrative example we analyze the distribution of the LLR at the output of a decoder relative to a 32-state rate-1 RCC with generator polynomial $G=75$ and feedback polynomial $H=53$-this will correspond to the outer code of the the code $\mathrm{SCCC}_{\mathrm{opt}}$ derived in Subsection IV-B. In Fig. 5, the actual pdf of the LLR at the decoder output is compared with the Gaussian approximating pdf, setting $\rho=0.9$ and considering two different values for the input SNR, namely 1 and 2. As one can see, the Gaussian approximation is very accurate. This particularly holds for negative values of the LLRs, i.e., in the region where the feedback provided to the other component decoder is negative. In this region, accuracy is of paramount importance, since the feedback tries to redirect the decoding trend of the other decoder.

As in usual density evolution for turbo decoding [23], one can graphically visualize the evolution of the SNRs of the messages exchanged between the decoders. In other words, the performance of a SCCC can be inferred, for given values of $\gamma_{b}$, $\rho$, and $\tilde{\rho}$ by graphically comparing the curves corresponding to (i) the output SNR of decoder DEC ${ }^{(i)}$ as a function (denoted 


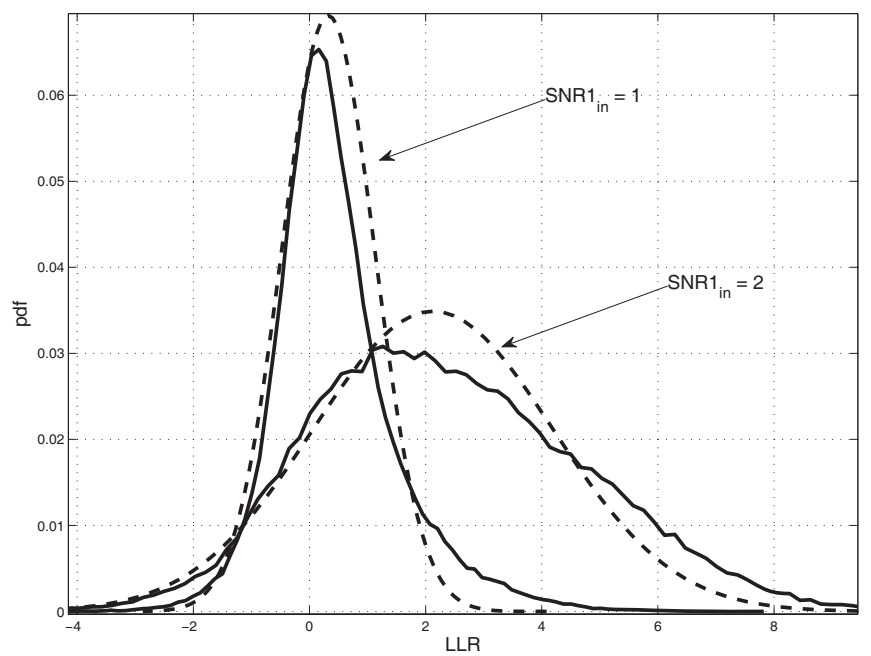

Fig. 5. Comparison between the actual pdf of the LLR at the output of a decoder, relative to a 32-state rate-1 RCC with generator polynomial $G(D)=$ 75 , and its Gaussian approximation. The parameter $\rho$ is set to 0.9 and two different values for the input SNR are considered.

as $Z_{\mathrm{i}}$ ) of its input $\mathrm{SNR}^{5}$ and (ii) the input SNR of decoder $\mathrm{DEC}^{(\mathrm{o})}$ as a function (denoted as $Z_{\mathrm{o}}^{-1}$ ) of its output SNR. In Fig. 6, we show the SNR curves $Z_{\mathrm{i}}$ and $Z_{\mathrm{o}}^{-1}$ for the two rate$1 / 4$ SCCCs introduced in Subsection III-B, setting $\rho=\tilde{\rho}=0.9$ (i.e., under the assumption of perfect reliability estimation) and $\gamma_{\mathrm{b}}=-5 \mathrm{~dB}$ in all cases. In the "RCC outer-RCC inner" case the curves are far from each other, i.e., the convergence to zero BEP is faster that in the "NRCC outer-RCC inner" case. This result confirms the conjecture, drawn in Subsection III-B, regarding the design of a good outer code, thus making the "RCC outer-RCC inner" SCCC the best. Moreover, it shows that the current density evolution-based design criterion leads to results consistent with those obtained with the union boundbased criterion.

\section{B. Case Study: Optimized Rate-1/2 Punctured SCCCs}

In this subsection, we apply the density evolution-based framework introduced in Subsection IV-A to select optimized rate-1/2 punctured SCCCs among a set of possible candidate SCCCs. We assume perfect reliability estimation, i.e., $\rho=\tilde{\rho}$. Moreover, according to the optimization conjecture discussed in Subsection IV-A, we consider as candidate SCCCs only those which have, as inner code, the rate-1/2 systematic RCC derived from the equivalent NRCC with maximum free distance shown in [19], and, as outer code, the rate-1/2 non systematic RCC derived according to the optimization framework proposed in Subsection III-A. Since the considered component $\mathrm{CCs}$ have rate $1 / 2$, the desired overall rate equal to $1 / 2$ can be obtained by puncturing the $\mathrm{CCs}$, thus obtaining different combinations of outer and inner coding rates. Denoting by $r^{(\mathrm{o})}$ and $r^{(\mathrm{i})}$ the outer and inner code rates, respectively, we consider four possible combinations of $r^{(\mathrm{o})}$ and $r^{(\mathrm{i})}$ leading to a rate-1/2 SCCC: (i) 1 and $1 / 2$; (ii) $3 / 4$ and $2 / 3$; (iii) $2 / 3$ and $3 / 4$; (iv) $1 / 2$ and 1 . Considering

\footnotetext{
${ }^{5}$ Note that the output/input SNR refer to the SNR of the input/output sequences.
}
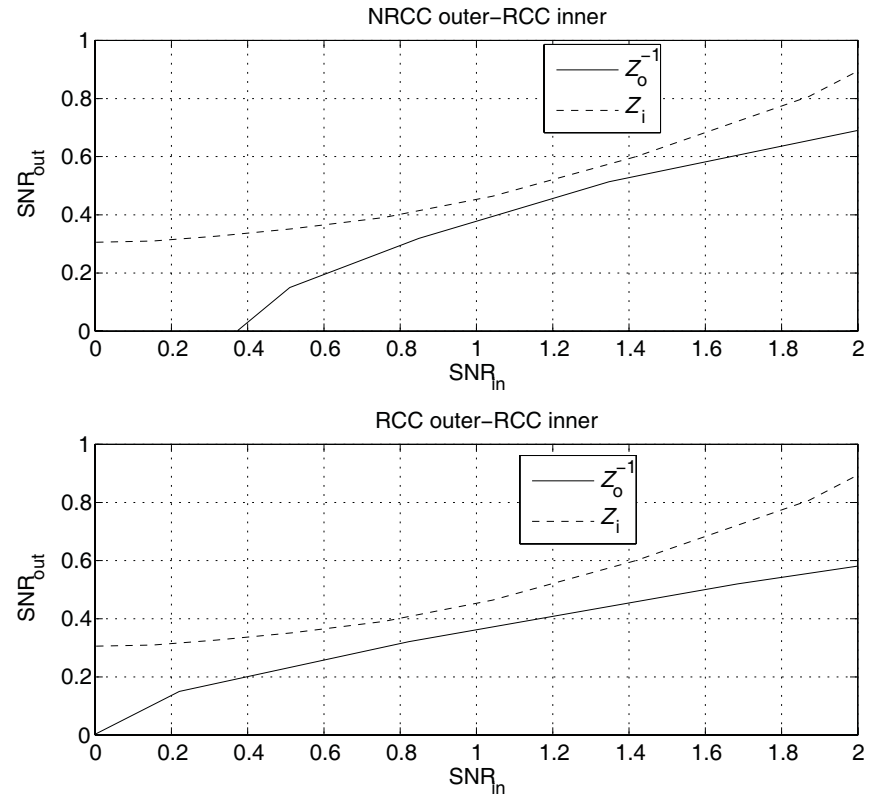

Fig. 6. Density evolution for three different rate-1/4 SCCCs. In all cases, $\rho=0.9$ and $\gamma_{\mathrm{b}}=-5 \mathrm{~dB}$.

a single rate-1/2 starting $\mathrm{CC}$, a puncturing pattern (PP) of minimum period leading to a rate- $p / q \mathrm{CC}$ is determined by a $2 \times p$ matrix with $q$ ones and $2 p-q$ zeros: this yields an overall set of $\left(\begin{array}{c}2 p \\ q\end{array}\right)$ possible puncturing patterns. Hence, the considered possible rates of a punctured CC (either outer or inner), namely $1,2 / 3$, and $3 / 4$, correspond to $\left(\begin{array}{l}2 \\ 1\end{array}\right),\left(\begin{array}{l}4 \\ 3\end{array}\right)$, and $\left(\begin{array}{l}6 \\ 4\end{array}\right)$ puncturing patterns. On the other hand, if the rate remains $1 / 2$ there is only $\left(\begin{array}{l}2 \\ 2\end{array}\right)=1$ possibility, i.e., the non-punctured starting CC. By fixing the values of $\rho$ and $\gamma_{b}$, it is possible to draw the $\mathrm{SNR}_{\mathrm{in}}-\mathrm{SNR}_{\text {out }}$ curves, relative to the inner and outer component CCs (curves $Z_{\mathrm{i}}$ and $Z_{\mathrm{o}}^{-1}$, respectively), according to the procedure described in Subsection IV-A. On the basis of the combinatorial considerations just carried out and taking into account all rate combinations, the overall number of pairs of inner-outer curves (i.e., of possible SCCCs) to be considered is $\left(\begin{array}{l}2 \\ 1\end{array}\right)\left(\begin{array}{l}2 \\ 2\end{array}\right)+\left(\begin{array}{l}6 \\ 4\end{array}\right)\left(\begin{array}{l}4 \\ 3\end{array}\right)+\left(\begin{array}{l}4 \\ 3\end{array}\right)\left(\begin{array}{l}6 \\ 4\end{array}\right)+\left(\begin{array}{l}2 \\ 2\end{array}\right)\left(\begin{array}{l}2 \\ 1\end{array}\right)=124$. For each SCCC, we define as $\gamma_{b}^{\text {th }}$ the minimum SNR at which its associated curves $Z_{\mathrm{i}}$ and $Z_{\mathrm{o}}^{-1}$ do not touch, i.e., the SNR associated with the "knee" of the BEP curve: the lower $\gamma_{b}^{\text {th }}$, the better the SCCC. Since different encoders may have different optimal puncturing patterns [24], [25], for various pairs of constraint lengths $v^{(\mathrm{i})}$ and $v^{(\mathrm{o})}$ of the component inner and outer CCs, we carry out an exhaustive search over all possible PP. The obtained results, obtained setting $\rho=0.9$, are shown in Table II. As one can see, for all possible values of constraint lengths, the optimal PPs (given by $2 \times 1$ matrices), denoted as $P P_{\mathrm{opt}}^{(\mathrm{o})}$ and $P P_{\mathrm{opt}}^{(\mathrm{i})}$, correspond to $r^{(\mathrm{o})}=1$ and $r^{(\mathrm{i})}=1 / 2$. The overall best SCCC, denoted as $\mathrm{SCCC}_{\mathrm{opt}}$, is the one constituted by: (i) the non-systematic 32-state $\left(v^{(\mathrm{o})}=5\right)$ rate-1 outer RCC with generator polynomial $G=75$ and feedback polynomial $H=53$; (ii) the systematic 4-state $\left(v^{(\mathrm{i})}=2\right)$ rate$1 / 2$ inner RCC with generator polynomials $G^{(1)}=5, G^{(2)}=7$ and feedback polynomial $H=7$. Our results (not shown here for lack of space) show that this SCCC remains the best for 
TABLE II

OPTIMIZED PUNCTURING PATTERNS $\left(P P_{\mathrm{opt}}^{(\mathrm{o})}, P P_{\mathrm{opt}}^{(\mathrm{i})}\right)$ AND CORRESPONDING SNR THRESHOLD $\gamma_{\mathrm{b}}^{\text {th }}$ AS FUNCTIONS OF THE OUTER AND INNER ENCODERS' MEMORIES $\left(v^{(\mathrm{o})}, v^{(\mathrm{i})}\right)$. IN ALL CASES, $\tilde{\rho}=\rho=0.9$.

\begin{tabular}{|c|c|c|c|c|}
\hline$v^{(\mathrm{o})}$ & $v^{(\mathrm{i})}$ & $P P_{\mathrm{opt}}^{(\mathrm{o})}$ & $P P_{\mathrm{opt}}^{(\mathrm{i})}$ & $\gamma_{\mathrm{b}}^{\text {th }}[\mathrm{dB}]$ \\
\hline 2 & 3 & $\begin{array}{l}0 \\
1\end{array}$ & $\begin{array}{l}1 \\
1\end{array}$ & -2.7 \\
\hline 2 & 4 & $\begin{array}{l}0 \\
1\end{array}$ & $\begin{array}{l}1 \\
1\end{array}$ & -2.45 \\
\hline 2 & 5 & $\begin{array}{l}0 \\
1\end{array}$ & $\begin{array}{l}1 \\
1\end{array}$ & -2.9 \\
\hline 3 & 2 & $\begin{array}{l}0 \\
1\end{array}$ & $\begin{array}{l}1 \\
1\end{array}$ & -2.5 \\
\hline 3 & 3 & $\begin{array}{l}0 \\
1\end{array}$ & $\begin{array}{l}1 \\
1\end{array}$ & -3 \\
\hline 3 & 4 & $\begin{array}{l}0 \\
1\end{array}$ & $\begin{array}{l}1 \\
1\end{array}$ & -2.85 \\
\hline 3 & 5 & $\begin{array}{l}0 \\
1\end{array}$ & $\begin{array}{l}1 \\
1\end{array}$ & -2.85 \\
\hline 4 & 2 & $\begin{array}{l}0 \\
1\end{array}$ & $\begin{array}{l}1 \\
1\end{array}$ & -1.9 \\
\hline 4 & 3 & $\begin{array}{l}0 \\
1\end{array}$ & $\begin{array}{l}1 \\
1\end{array}$ & -3 \\
\hline 4 & 4 & $\begin{array}{l}0 \\
1\end{array}$ & $\begin{array}{l}1 \\
1\end{array}$ & -2.85 \\
\hline 4 & 5 & $\begin{array}{l}0 \\
1\end{array}$ & $\begin{array}{l}1 \\
1\end{array}$ & -2.75 \\
\hline 5 & 2 & $\begin{array}{l}0 \\
1\end{array}$ & $\begin{array}{l}1 \\
1\end{array}$ & -3.2 \\
\hline 5 & 3 & $\begin{array}{l}0 \\
1\end{array}$ & $\begin{array}{l}1 \\
1\end{array}$ & -3 \\
\hline 5 & 4 & $\begin{array}{l}0 \\
1\end{array}$ & $\begin{array}{l}1 \\
1\end{array}$ & -2.9 \\
\hline 5 & 5 & $\begin{array}{l}0 \\
1\end{array}$ & $\begin{array}{l}1 \\
1\end{array}$ & -2.8 \\
\hline
\end{tabular}

large values of $\rho$, namely $\rho \geq 0.8$.

The obtained SCCC design guidelines are in contrast with "classical" design criteria in the absence of API [22]. The intuition behind this result consists of the fact that the outer CC can be kept "less powerful" (i.e., $r^{(0)}=1$ and high free distance), since it will rely on the presence of API. For a given SCCC rate, this allows to correspondingly make the inner $\mathrm{CC}$ more powerful (i.e., to reduce $\left.r^{(\mathrm{i})}\right)$. On the other hand, reducing the rate of the outer $\mathrm{CC}$ makes the impact of the API less relevant and leaves the inner $\mathrm{CC}$ weaker, thus leading to an overall performance degradation for the SCCC. In order to provide an illustrative example, we compare the $Z_{\mathrm{i}}$ and $Z_{\mathrm{o}}^{-1}$ curves of the component $\mathrm{CCs}$ of $\mathrm{SCCC}_{\mathrm{opt}}$ with those of the SCCC with the same memories for the component CCs (namely, $v^{(\mathrm{i})}=2$ and $v^{(\mathrm{o})}=5$ ) and with coding rates $r^{(\mathrm{o})}=$ $2 / 3$ and $r^{(\mathrm{i})}=3 / 4$. The latter SCCC is denoted as $\mathrm{SCCC}_{\mathrm{B}}$. The obtained results are shown in Fig. 7, where $\tilde{\rho}=\rho=0.9$ and $\gamma_{\mathrm{b}}=-2 \mathrm{~dB}$. In this figure, it is shown clearly that considering a lower rate for the outer $\mathrm{CC}$ moves, for sufficiently large values of $\mathrm{SNR}_{\mathrm{in}}$, the $Z_{\mathrm{o}}^{-1}$ (lower) curve down. Even though this is positive, the associated increase of the rate of the inner CC moves the $Z_{\mathrm{i}}$ (upper) curve even more down. Overall, reducing the rate of the outer $\mathrm{CC}$ and, correspondingly, increasing the rate of the inner $\mathrm{CC}$ closes the "tunnel" between $Z_{\mathrm{i}}$ and $Z_{\mathrm{o}}^{-1}$, i.e., increases the threshold SNR. In other words, since the outer CC is "helped" by the API, our results show that it is more convenient to make the inner $\mathrm{CC}$ more robust (i.e., reducing its rate).

The validity of the proposed design criterion is confirmed

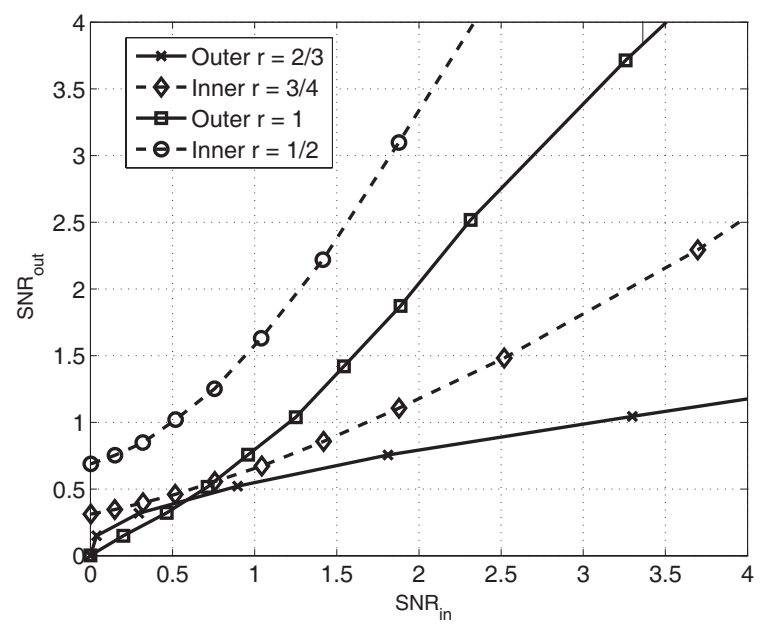

Fig. 7. Graphical density evolution considering two SCCCs with $v^{(\mathrm{i})}=2$ and $v^{(\mathrm{o})}=5$ : $\operatorname{SCCC}_{\mathrm{opt}}\left(r^{(\mathrm{o})}=1\right.$ and $\left.r^{(\mathrm{i})}=1 / 2\right)$ and $\operatorname{SCCC}_{\mathrm{B}}\left(r^{(\mathrm{o})}=2 / 3\right.$ and $\left.r^{(\mathrm{i})}=3 / 4\right)$. In all cases, $\tilde{\rho}=\rho=0.9$ and $\gamma_{\mathrm{b}}=-2 \mathrm{~dB}$.

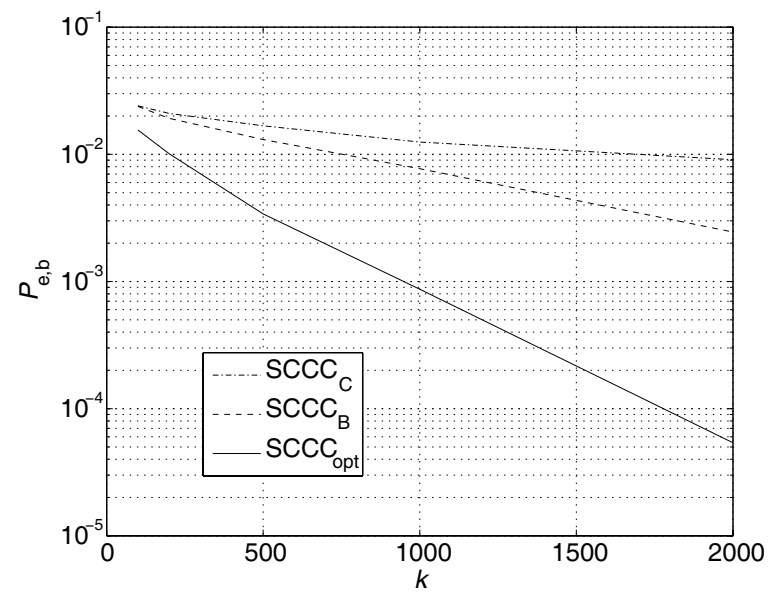

Fig. 8. BEP, as a function of the information sequence length $k$, considering various SCCCs. In all cases, $\gamma_{\mathrm{b}}=-2 \mathrm{~dB}$ and $\rho=0.9$.

also by the results presented in Fig. 8 , where $P_{\mathrm{b}, \mathrm{e}}$ is shown, as a function of the information sequence length $k$, considering $\gamma_{\mathrm{b}}=-2 \mathrm{~dB}$ and $\rho=0.9$. Together with $\mathrm{SCCC}_{\mathrm{opt}}$ and $\mathrm{SCCC}_{\mathrm{B}}$, we consider also the SCCC, denoted as $\mathrm{SCCC}_{\mathrm{C}}$, derived from $\mathrm{SCCC}_{\mathrm{B}}$ by replacing the outer RCC with its corresponding non-recursive version. As one can see, the proposed design rule becomes even more appealing for increasing codeword lengths.

In order to verify the predictions of the density evolutionbased design criterion on the optimum SCCC structure, we now compare, through Monte Carlo simulations, the performance of $\mathrm{SCCC}_{\mathrm{opt}}$ and $\mathrm{SCCC}_{\mathrm{B}}$. All simulated rate-1/2 SCCCs use random interleavers and the length of the information sequence is $k=262144$. Each BEP value is obtained by considering 400 information packets. ${ }^{6}$ The number of iterations used in the decoder is 50. In Fig. 9, the BEP is shown as a function of the SNR, considering perfect reliability estimation (i.e., $\tilde{\rho}=\rho$ ) and three possible values of $\rho: 0.8,0.9$, and 0.95 .

\footnotetext{
${ }^{6}$ The simulation set-up guarantees a reliable estimation of a BEP of the order of $10^{-5}$ with more than 1000 errors.
} 


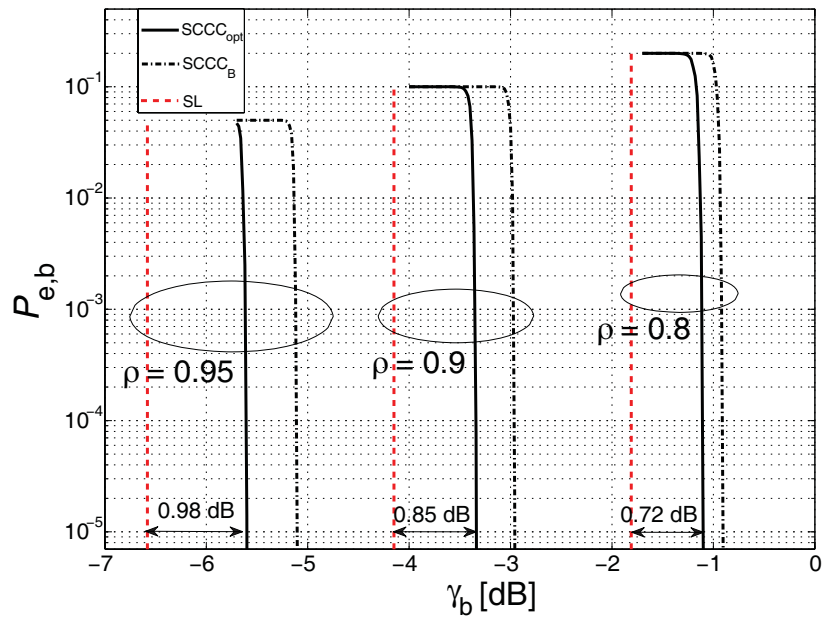

Fig. 9. BEP, as a function of the SNR, considering two rate-1/2 SCCCs: $\mathrm{SCCC}_{\text {opt }}$ and $\mathrm{SCCC}_{\mathrm{B}}$. In all cases, $\tilde{\rho}=\rho$. Three possible values of $\rho$ are considered: $0.8,0.9$, and 0.95 .

As expected, $\mathrm{SCCC}_{\mathrm{opt}}$ offers the best "waterfall" performance for all values of $\rho$. In order to highlight the excellent behavior of $\mathrm{SCCC}_{\mathrm{opt}}$, in Fig. 9 we also show the gaps, with respect to the corresponding SL SNRs, for the three considered values of $\rho$. In particular, owing to the definition of $\gamma_{b}$ (in Section II) as the SNR at the receiver and taking into account that $1 / 2$ is the considered code rate, the SNR (dimension: [dB]) corresponding to the SL can be written as $10 \log _{10}\left(2^{H_{\mathrm{e}}(\rho)}-1\right)$, where $H_{\mathrm{e}}(\rho) \triangleq-\rho \log _{2} \rho-(1-\rho) \log _{2}(1-\rho)$. The gap of SCCC $_{\text {opt }}$ from the corresponding SL (for a given value of $\rho$ ) is then evaluated as the SNR difference at a BEP equal to $10^{-5}$. For the three considered values of $\rho(0.8,0.9$, and 0.95$)$ the SL SNRs are $-1.87 \mathrm{~dB},-4.15 \mathrm{~dB}$, and $-6.58 \mathrm{dBs}$, respectively, and the corresponding gaps of $\mathrm{SCCC}_{\mathrm{opt}}$ are $0.72 \mathrm{~dB}, 0.85 \mathrm{~dB}$, and $0.98 \mathrm{~dB}$. Considering the cases with $\rho=0.8$ and $\rho=0.9$, one can directly compare the performance of $\mathrm{SCCC}_{\text {opt }}$ with that of the non-systematic turbo code (NSTC) proposed in [16] for the same information sequence length. In particular, the NSTC presented in [16], which, to the best of our knowledge, is the coding scheme which guarantees the shortest gap from the SL, allows to reach, for $\rho$ equal to 0.8 and 0.9 , gaps equal to $0.87 \mathrm{~dB}$ and $1.05 \mathrm{~dB}$, respectively. Therefore, one can conclude that the proposed $\mathrm{SCCC}_{\mathrm{opt}}$ allows to outperform previously presented coding schemes for medium-to-(very) high values of $\rho$. Note that if API were available at the encoder side, then our optimization approach could be extended to encompass the use of proper energy allocation techniques, such as those considered in [26].

Finally, we verify the behavior of the proposed $\mathrm{SCCC}_{\mathrm{opt}}$ in the presence of imperfect reliability estimation. In order to do this, $\gamma_{b}^{\text {th }}$ (i.e., the minimum value of $\gamma_{b}$ required for decoding convergence) is evaluated it both through simulations and through the proposed density evolution-based framework. More specifically: with the simulation-based approach, $\gamma_{b}^{\text {th }}$ is evaluated as the value of $\gamma_{b}$ which allows to achieve a BEP equal to $10^{-5}$; with the the density evolution-based approach, $\gamma_{b}^{\text {th }}$ corresponds to the minimum value of $\gamma_{b}$ for which the curves $Z_{\mathrm{i}}$ and $Z_{\mathrm{o}}^{-1}$ do not touch. In Fig. $10, \gamma_{\mathrm{b}}^{\text {th }}$ of $\mathrm{SCCC}_{\mathrm{opt}}$

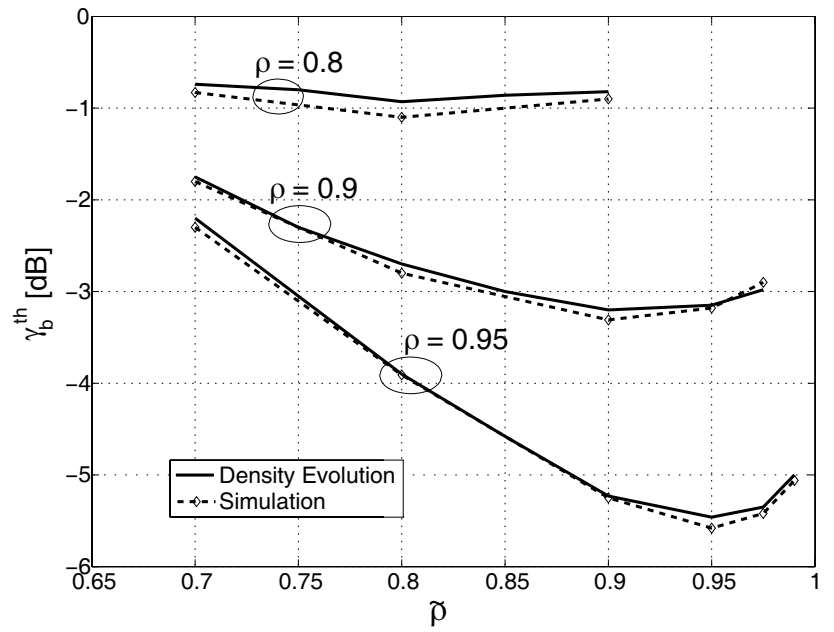

Fig. 10. $\gamma_{b}^{\text {th }}$, as a function of $\tilde{\rho}$, computed through simulations (dashed lines with symbols) and through density evolution (solid lines). Three possible values of $\rho$ are considered: $0.8,0.9$, and 0.95 .

is shown as a function of $\tilde{\rho}$, considering three possible values for $\rho: 0.8,0.9$, and 0.95 . As one can observe, the density evolution-based analysis allows to predict accurately the performance of $\mathrm{SCCC}_{\mathrm{opt}}$, even in the case of imperfect estimation of $\rho$ (i.e., $\tilde{\rho} \neq \rho$ ). Obviously, the lowest value of $\gamma_{\mathrm{b}}^{\text {th }}$ is obtained, in each case, for $\tilde{\rho}=\rho$. Moreover, it can be observed that $\mathrm{SCCC}_{\text {opt }}$ guarantees a limited increase of $\gamma_{b}$ even in the presence of a moderate reliability estimation error. In other words, the performance of the proposed optimized SCCCing scheme is robust against estimation errors.

\section{CONCLUSiOnS}

In this paper, we have first derived a BEP-based analytical framework for the design of optimized CCs in the presence of API at the decoder. This BEP union bound-based framework has then be extended to encompass the design of optimized SCCCs. In particular, the optimized SCCCs use, as outer component codes, the previously optimized CCs. Since the BEP union bound-based approach applies to the BEP floor of SCCCs, in order to optimize their design in the waterfall region we have proposed a density evolution-based optimization approach, which confirms the results obtained with the BEP union bound-based framework. Experimental results show that the proposed SCCCing schemes allow to noticeably outperform the performance of turbo coding schemes optimized for scenarios where no API is available at the decoder. Moreover, in the presence of relevant API the proposed optimized SCCC allows to approach the SL more than any previously proposed channel coding scheme.

\section{ACKNOWLEDGMENTS}

The authors would like to thank the anonymous Reviewers of this paper, whose insightful comments helped to improve the quality of the paper substantially. 


\section{REFERENCES}

[1] J. Hagenauer, "Source-controlled channel decoding," IEEE Trans. Commun., vol. 43, no. 9, pp. 2449-2457, Sep. 1995.

[2] M. Park and D. J. Miller, "Joint source-channel decoding for variable length encoded data by exact and approximate MAP sequence estimation," IEEE Trans. Commun., vol. 48, no. 1, pp. 1-6, Jan. 2000.

[3] C. Lamy and O. Pothier, "Reduced complexity maximum a posteriori decoding of variable-length codes," in Proc. IEEE Global Telecommun. Conf., San Antonio, TX, USA, Nov. 2001, pp. 1410-1413.

[4] M. Jeanne, J. C. Carlach, and P. Siohan, "Joint source-channel decoding of variable-length codes for convolutional codes and turbo codes," IEEE Trans. Commun., vol. 53, no. 1, pp. 10-15, Jan. 2005.

[5] X. Liu, S. N. Koh, and T. H. Cheng, "Improved bit-based joint sourcechannel decoding of variable length codes," IEEE Commun. Lett., vol. 11 , no. 6, pp. 516-518, June 2007.

[6] P. M. Crespo, E. Loyo, J. Del Ser, and C. J. Mitchell, "Source controlled modulation scheme for sources with memory," in Proc. IEEE Intern. Conf. on Commun., Glascow, Scotland, June 2007, pp. 2619-2624.

[7] G.-C. Zhu and F. Alajaji, "Joint source-channel turbo coding for binary Markov sources," IEEE Trans. Wireless Commun., vol. 5, no. 5, pp. 1065-1075, May 2006.

[8] L. Pu, Z. Wu, A. Bilgin, M. W. Marcellin, and A. Bilgin, "LDPCbased iterative joint source-channel decoding for JPEG2000," IEEE Trans. Image Processing, vol. 16, no. 2, pp. 577-581, Feb. 2007.

[9] I. F. Akyildiz, W. Su, Y. Sankarasubramaniam, and E. Caryirci, "A survey on sensor networks," IEEE Commun. Mag., vol. 40, no. 8, pp. 102-114, Aug. 2002.

[10] J. Garcia-Frias and Y. Zhao, "Compression of correlated binary sources using turbo codes," IEEE Commun. Lett., vol. 5, no. 10, pp. 417-419, Oct. 2001.

[11] W. Zhao and J. Garcia-Frias, "Joint estimation and compression of correlated nonbinary sources using punctured turbo codes," IEEE Trans. Commun., vol. 53, no. 3, pp. 385-390, Mar. 2005.

[12] J. Garcia-Frias, Y. Zhao, and W. Zhong, "Turbo-like codes for transmission of correlated sources over noisy channels," IEEE Signal Process. Mag., vol. 24, no. 5, pp. 58-66, Sep. 2007.

[13] F. Daneshgaran, M. Laddomada, and M. Mondin, "Iterative joint channel decoding of correlated sources employing serially concatenated convolutional codes," IEEE Trans. Inf. Theory, vol. 51, no. 7, pp. 2721-2731, Aug. 2005.

[14] J. Muramatsu, T. Uyematsu, and T. Wadayama, "Low-density paritycheck matrices for coding of correlated sources," IEEE Trans. Inf. Theory, vol. 51, no. 10 , pp. 3645-3654, Oct. 2005.

[15] S. Shamai and S. Verdù, "The empirical distribution of good codes," IEEE Trans. Inf. Theory, vol. 43, no. 3, pp. 836-846, May 1997.

[16] G.-C. Zhu, F. Alajaji, J. Bajesy, and P. Mitran, "Transmission of nonuniform memoryless sources via nonsystematic turbo codes," IEEE Trans. Commun., vol. 52, no. 8, pp. 1344-1354, Aug. 2004.

[17] A. Abrardo, "Performance bounds and codes design criteria for channel decoding with a-priori information," IEEE Trans. Wireless Commun., vol. 8, no. 2, pp. 608-612, Feb. 2009.

[18] B. Sklar, editor, Digital Communications: Fundamentals and Applications. IEEE Press, 2001.

[19] J. G. Proakis, Digital Communications, 4th edition. McGraw-Hill, 2001.

[20] J.-J. Chang, D.-J. Hwang, and M. C. Lin, "Some extended results on the search for good convolutional codes," IEEE Trans. Inf. Theory, vol. 43, no. 5, pp. 1682-1697, Sep. 1997.

[21] S. Benedetto, R. Garello, and G. Montorsi, "A search for good convolutional codes to be used in the construction of turbo codes," IEEE Trans. Commun., vol. 46, no. 9, pp. 1101-1105, Sep. 1998.

[22] S. Benedetto, D. Divsalar, G. Montorsi, and F. Pollara, "Serial concatenation of interleaved codes: performance analysis, design, and iterative decoding," IEEE Trans. Inf. Theory, vol. 44, no. 3, pp. 909-926, May 1998.

[23] D. Divsalar, S. Dolinar, and F. Pollara, "Iterative turbo decoder analysis based on density evolution," IEEE J. Sel. Areas Commun., vol. 19, no. 5, pp. 891-907, May 2001.

[24] G. Begin, D. Haccoun, and C. Paquin, "Further results on high-rate punctured convolutional codes for Viterbi and sequential decoding," IEEE Trans. Commun., vol. 38, no. 11, pp. 1922-1928, Nov. 1990.

[25] A. Graell i Amat, G. Montorsi, and S. Benedetto, "Design and decoding of optimal high-rate convolutional codes," IEEE Trans. Inf. Theory, vol. 50, no. 5, pp. 867-881, May 2004.
[26] F. Cabarcas, R. D. Souza, and J. Garcia-Frias, "Turbo coding of strongly non-uniform memoryless sources with unequal energy allocation and PAM signaling," IEEE Trans. Signal Process., vol. 54, no. 5, pp. 1942 1946, May 2006.

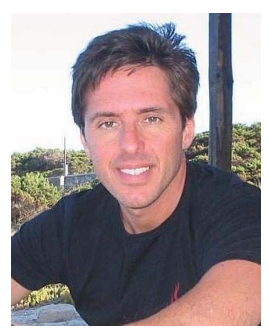

Andrea Abrardo (http://www.dii.unisi.it) graduated in Electronic Engineering at the University of Florence, Italy, in 1993. Since January 1994 to November 1994 he has worked in the Image Processing and Communications Laboratory of the Department of Electronic Engineering of the University of Florence collaborating with the Tuscany Region for the development of broad-band networks infrastructures. Since November 1994 to October 1997 he attended the Ph.D. at the same Department of Electronic Engineering. In June 1998 he got the Ph.D. degree discussing a Thesis on "Web based Teleradiology systems," In 1998 he joined the Department of Informatic Engineering of the University of Siena, Italy, as a Researcher. His current position at the Department of Informatic Engineering of the University of Siena is Associate Professor. His research interests are in the field of resource allocation strategies for wireless networks, with en emphasis on ad-hoc and sensor networks. He his currently teaching Digital Transmission and Mobile Communications at the University of Siena. As a result of his research activity he has published more then 90 works on international journals and conferences.

During his research activity, he has been involved in several National and European Projects. In particular, he has been coordinator of the National project "Radio resource management and localization of users for multimedia vehicular applications," within the CNR-Agenzia2000 programme. Moreover, he has been local coordinator of the three years (2002-2005) National FIRB project "Reconfigurable Platforms for wide band radiomobile communications." The research activity in this context was in particolar aimed at the definition and practical implementation of dynamic radio resource allocation strategies for OFDMA wireless systems. Eventually, he is WP leader in the ST@RT project following the activity: "Development of a wireless sensor network for the monitoring of historical and artistic edifices."

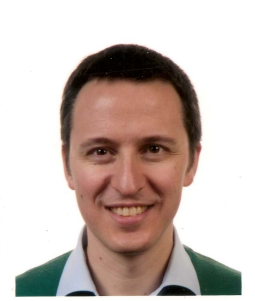

Gianluigi Ferrari (http://www.tlc.unipr.it/ferrari) was born in Parma, Italy, in 1974. He received his "Laurea" and $\mathrm{PhD}$ degrees from the University of Parma, Italy, in 1998 and 2002, respectively. Since 2002, he has been with the University Parma, where he currently is an Associate Professor of Telecommunications. He was a visiting researcher at USC (Los Angeles, CA, USA, 2000-2001), CMU (Pittsburgh, PA, USA, 2002-2004), KMITL (Bangkok, Thailand, 2007), and ULB (Bruxelles, Belgium, 2010). Since 2006, he has been the Coordinator of the Wireless Ad-hoc and Sensor Networks (WASN) Lab in the Department of Information Engineering of the University of Parma.

As of today he has published more than 140 papers in leading international journals and conferences. He is coauthor of a few books, including Detection Algorithms for Wireless Communications, with Applications to Wired and Storage Systems (Wiley: 2004), Ad Hoc Wireless Networks: A Communication-Theoretic Perspective (Wiley: 2006), LDPC Coded Modulations (Springer: 2009), and Sensor Networks with IEEE 802.15.4 Systems: Distributed Processing, MAC, and Connectivity (Springer: 2011). He edited the book Sensor Networks: where Theory Meets Practice (Springer: 2010). His research interests include digital communication systems analysis and design, wireless ad hoc and sensor networking, adaptive digital signal processing, and information theory.

Dr. Ferrari is a co-recipient of a best student paper award at the 2006 International Workshop on Wireless Ad hoc Networks (IWWAN'06). He acts as a frequent reviewer for many international journals and conferences. $\mathrm{He}$ acts also as a technical program member for many international conferences. He currently serves on the Editorial Boards of The Open Electrical and Electronic Engineering (TOEEJ) Journal (Bentham Publishers), the International Journal of RF Technologies: Research and Applications (Taylor \& Francis), and the International Journal of Future Generation Communication and Networking (SERSC: Science \& Engineering Research Support Center). He was a Guest Editor of the 2009 EURASIP JWCN Special Issue on "Dynamic Spectrum Access: From the Concept to the Implementation." 